\title{
The complete mitochondrial genome of parasitic nematode Camallanus cotti: extreme discontinuity in the rate of mitogenomic architecture evolution within the Chromadorea class
}

\author{
Hong Zou', Ivan Jakovlić ${ }^{1}$, Rong Chen ${ }^{2}$, Dong Zhang ${ }^{1,3} \mathbb{D}$, Jin Zhang ${ }^{2}$, Wen-Xiang Li ${ }^{i^{*}}$ and Gui-Tang Wang ${ }^{1}$
}

\begin{abstract}
Background: Complete mitochondrial genomes are much better suited for the taxonomic identification and phylogenetic studies of nematodes than morphology or traditionally-used molecular markers, but they remain unavailable for the entire Camallanidae family (Chromadorea). As the only published mitogenome in the Camallanina suborder (Dracunculoidea superfamily) exhibited a unique gene order, the other objective of this research was to study the evolution of mitochondrial architecture in the Spirurida order. Thus, we sequenced the complete mitogenome of the Camallanus cotti fish parasite and conducted structural and phylogenomic comparative analyses with all available Spirurida mitogenomes.

Results: The mitogenome is exceptionally large (17,901 bp) among the Chromadorea and, with 46 (pseudo-) genes, exhibits a unique architecture among nematodes. Six protein-coding genes (PCGs) and six tRNAs are duplicated. An additional (seventh) tRNA (Trp) was probably duplicated by the remolding of tRNA-Ser2 (missing). Two pairs of these duplicated PCGs might be functional; three were incomplete and one contained stop codons. Apart from Ala and Asp, all other duplicated tRNAs are conserved and probably functional. Only 19 unique tRNAs were found. Phylogenomic analysis included Gnathostomatidae (Spirurina) in the Camallanina suborder.

(Continued on next page)
\end{abstract}

\footnotetext{
* Correspondence: liwx@ihb.ac.cn

${ }^{1}$ Key Laboratory of Aquaculture Disease Control, Ministry of Agriculture, and

State Key Laboratory of Freshwater Ecology and Biotechnology, Institute of

Hydrobiology, Chinese Academy of Sciences, Wuhan 430072, People's

Republic of China

Full list of author information is available at the end of the article
} 
(Continued from previous page)

Conclusions: Within the Nematoda, comparable PCG duplications were observed only in the enoplean Mermithidae family, but those result from mitochondrial recombination, whereas characteristics of the studied mitogenome suggest that likely rearrangement mechanisms are either a series of duplications, transpositions and random loss events, or duplication, fragmentation and subsequent reassembly of the mitogenome. We put forward a hypothesis that the evolution of mitogenomic architecture is extremely discontinuous, and that once a long period of stasis in gene order and content has been punctuated by a rearrangement event, such a destabilised mitogenome is much more likely to undergo subsequent rearrangement events, resulting in an exponentially accelerated evolutionary rate of mitogenomic rearrangements. Implications of this model are particularly important for the application of gene order similarity as an additive source of phylogenetic information. Chromadorean nematodes, and particularly Camallanina clade (with C. cotti as an example of extremely accelerated rate of rearrangements), might be a good model to further study this discontinuity in the dynamics of mitogenomic evolution.

Keywords: Gene order rearrangement, Gene duplication, tRNA duplication, Incomplete tRNA set, tRNA remolding, TDRL, Pseudogenes, Mitochondrial phylogenomics, Spirurida, Camallanina

\section{Background}

Metazoan invertebrate phylum Nematoda is likely to comprise as much as $90 \%$ of all living multicellular organisms [1-3]. Because of the parasitic lifestyles of many nematodes, which cause numerous human diseases and large financial losses to agriculture and livestock rearing, as well as their use as biodiversity indicators and model organisms (e.g. Caenorhabditis), nematodes have attracted ample scientific attention $[4,5]$. Prior to the application of molecular data, nematode taxonomy and phylogeny relied on a very limited number of morphological characters and ecological features. This, along with the absence of informative fossil records $[5,6]$ and pervasive convergent evolution, has resulted in very poorly resolved and often inconsistent classification systems $[2,5,7]$. Thus, molecular data are much better suited for identification and phylogenetic studies of nematodes. The most often used genetic marker for this type of studies in nematodes is $18 \mathrm{~S}$ rRNA (or nSSU) $[1,2,5]$. However, since the number of nematode taxa will soon exceed the number of nucleotides in this gene, it can be safely argued that even the theoretical resolving power of this approach is insufficient for the task [3]. Thus a marker with much higher resolving power shall have to be adopted by future studies. Complete mitogenomes appear as a strong candidate, as they can provide a phylogenetic resolution superior to the traditionally used molecular markers and precise divergence date estimates, and thus are becoming the marker of choice for the resolution of taxonomic controversies [2, 8-13].

The mitochondrion is a eukaryotic organelle descended about 1,000,000,000 years ago from an $\alpha$ proteobacterium [14, 15], whose major function in the organism is energy production. Mitochondria contain their own genomes with a modified genetic code, which are usually very compact (no introns, little intergenic DNA, and most genes show signs of selection for small size) and highly conserved in terms of gene content and organisation [10, 16-18]. Mitogenomes of nematodes, however, are characterized by relatively frequent gene rearrangements $[2,7,13]$, unique initiation codons $[19,20]$, fast nucleotide substitution rate, unconventional tRNAs [10], and sometimes even unique organisation [21,22], which makes them a promising model for studying the mechanisms of mitochondrial gene rearrangements and genome architecture evolution [9]. So far, however, the main obstacle to their broad application has been a limited availability of sequenced mitochondrial genomes. Even though the number of complete mitogenomes deposited in public databases has grown exponentially during the last few years, many taxonomic categories remain poorly or not at all represented.

Among the non-represented taxa is the entire Camallanoidea superfamily (Camallanina suborder, Spirurida order, Chromadorea class). Spirurida order is composed of Spirurina and Camallanina suborders, the latter of which contains only Camallanoidea and Dracunculoidea superfamilies. Camallanidae (the only Camallanoidea family) are almost globally-distributed gastrointestinal parasites of poikilothermic vertebrates [1]. Only two Camallanus species parasitising Chinese freshwater fish are currently recognised: $C$. cotti and $C$. hypophthalmichthys [23]. The former, C. cotti (Fujita 1927; synonyms: C. zacconis Li 1941 and C. fotedari Raina \& Dhar 1972) parasitises a large number of fish species, mostly belonging to Cypriniformes, Siluriformes and Perciformes orders [24]. Although native to Asia, as a result of the trade of ornamental fishes and the introduction of various poeciliids for mosquito control, it has become almost globally distributed during the last few decades $[24,25]$. Its cosmopolitan dispersal, relatively high pathogenicity [24] and exceptional life history flexibility [25] have garnered ample scientific attention. 
Single molecular markers, such as internal transcribed spacer of ribosomal DNA, have been employed to infer the phylogenetic relationships of Camallanidae [1, 23], but due to previously described limitations of this approach, phylogeny of this family and the entire Camallanina clade is poorly understood. Sequencing of the complete mitochondrial genome of $C$. cotti, and the associated phylogenomic analysis using all available mitogenomes belonging to Spirurida order, are meant to address this problem. More importantly, as both mitochondrial genomes that are currently available for the entire Camallanina suborder, Philometroides sanguineus [26] and Dracunculus medinensis (unpublished), exhibit unique gene orders [26, 27], we hypothesised that this mitogenomic architecture might be idiosyncratic to the entire Camallanina suborder. To establish whether the unique Dracunculoidea architecture is shared with the sister superfamily Camallanoidea, and thus contribute to the understanding of the evolution of mitochondrial genomic architecture of nematodes, we have sequenced and characterised the mitogenome of $C$. cotti and compared it structurally to the available Spirurida mitogenomes.

\section{Results and discussion}

The mitochondrial genome of $C$. cotti possesses a large number of duplicated genes, which makes it structurally unique among the nematodes. The mitogenome is accessible from the GenBank under the accession number MF580344. To make sure that these duplicated sequences are not artefacts from our own PCR amplification or numts [28], we have extracted DNA from an additional $C$. cotti specimen and amplified, sequenced and assembled its entire genome anew. The sequences were identical, apart from the minor variations expected among individual mitogenomes [29], thus excluding the possibility of a mistake in the sequencing and assembly process. Unfortunately, these two nematodes were obtained from the same fish specimen, so we cannot directly exclude the possibility of this unique architecture being a lineage-specific phenomenon. If identical, or very similar, architecture is not observed in other Camallanidae species in the future, it would be of interest to sequence another mitogenome belonging to this species to confirm that the architecture is species-specific.

\section{Taxonomic identity and phylogeny}

Following proposed guidelines for validation of new mitogenomes [30], along with a phylogenetic analysis using almost the entire mitogenomic sequence, we have also conducted a barcoding identification assessment using all 99 Camallanidae cox 1 sequences available in the BOLD database [31]. The queried sequence was nested within the monophyletic Camallanus cotti clade (Additional file 1).

The two approaches (maximum-likelihood and Bayesian inference) used in this study to estimate the phylogenetic position of $C$. cotti within the Spirurida clade produced identical dendrogram topologies, so only the former is shown in Fig. 1. Statistical support values were very high, particularly in the BI analysis, where all posterior probability values were 1.0. In (partial) agreement with previous studies using both nSSU rRNA and mitochondrial phylogenomics approaches [1, 5, 27, 32]. Our results indicate that Spirurida order is divided into both morphologically [1] and genetically clearly defined Spirurina suborder, here comprised of (Physalopteridae + (Thelaziidae + (Gongylonematidae + (Setariidae [paraphyletic] + Onchocercidae)))), and a monophyletic

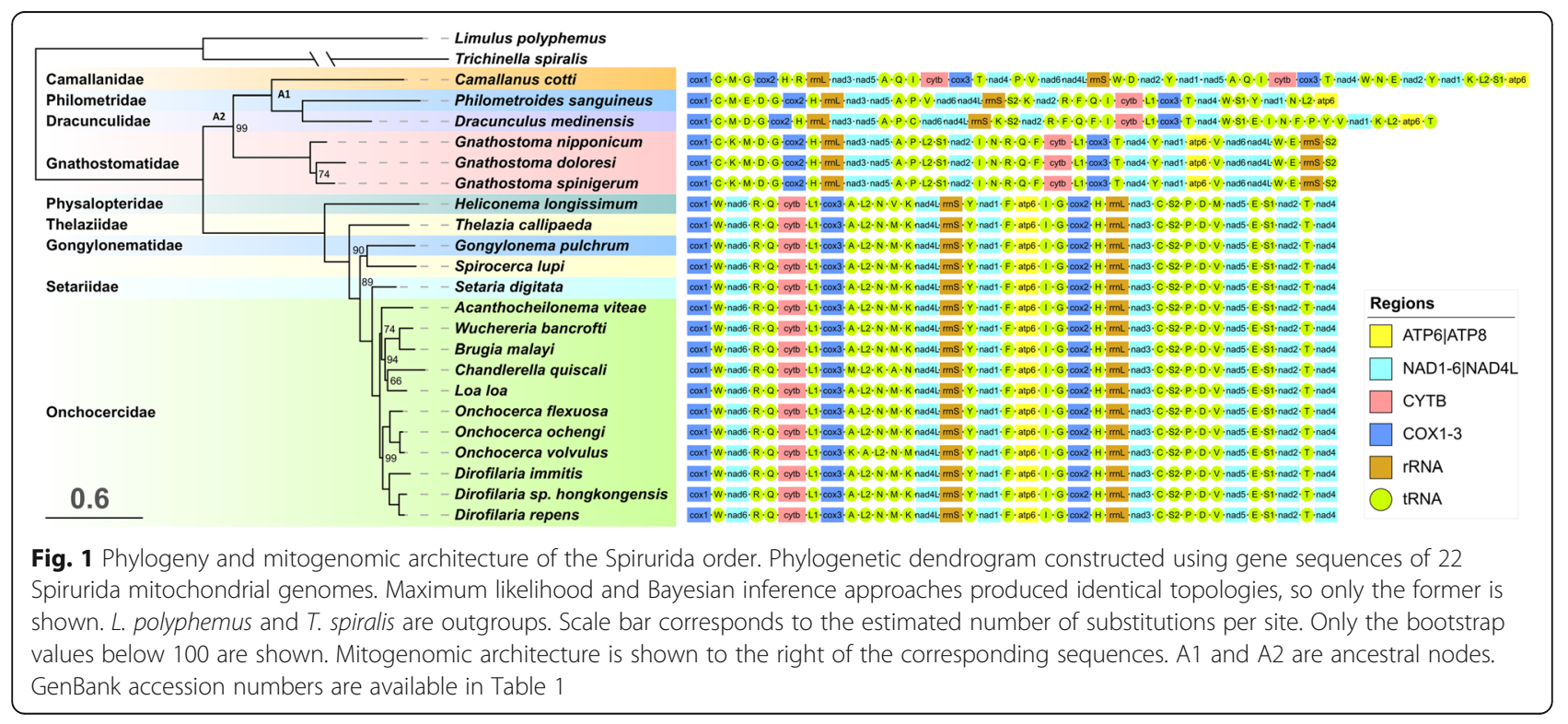


clade comprising (Gnathostomatidae + (Camallanidae + (Dracunculidae + Philometridae))), henceforth referred to as Camallanina suborder (Fig. 1, Table 1). Sister group relationship between the Camallanoidea superfamily (Camallanidae family) and Dracunculoidea superfamily (Philometridae and Dracunculidae in this study) is wellestablished [1, 5, 33, 34]. Paraphyly of Thelaziidae family, and particularly the problematic taxonomy of Spirocerca and Thelazia genera was also observed before $[1,5,27,35]$. A very similar topology of Spirurina clade, including the sister group relationship between

Table 1 Spirurida mitogenomes used for comparative and phylogenetic analyses in this study

\begin{tabular}{|c|c|c|c|}
\hline Species name & Family & Accession No. & Reference \\
\hline Camallanus cotti & Camallanidae & MF580344 & this study \\
\hline $\begin{array}{l}\text { Dracunculus } \\
\text { medinensis }\end{array}$ & Dracunculidae & NC_016019 & unpublished \\
\hline $\begin{array}{l}\text { Philometroides } \\
\text { sanguineus }\end{array}$ & Philometridae & NC_024931 & {$[26]^{b}$} \\
\hline $\begin{array}{l}\text { Acanthocheilonema } \\
\text { viteae }\end{array}$ & Onchocercidae & NC_016197 & {$[112]$} \\
\hline Brugia malayi & & NC_004298 & [113] \\
\hline Chandlerella quiscali & & NC_014486 & {$[112]$} \\
\hline Dirofilaria immitis & & NC_005305 & {$[62]$} \\
\hline Wuchereria bancrofti & & NC_016186 & {$[112]$} \\
\hline Loa loa & & NC_016199 & {$[112]$} \\
\hline $\begin{array}{l}\text { Dirofilaria sp. } \\
\text { 'hongkongensis' }\end{array}$ & & NC_031365 & {$[36]$} \\
\hline Dirofilaria repens & & NC_029975 & unpublished \\
\hline $\begin{array}{l}\text { Onchocerca } \\
\text { ochengi }\end{array}$ & & NC_031891 & unpublished \\
\hline $\begin{array}{l}\text { Onchocerca } \\
\text { volvulus }\end{array}$ & & NC_001861 & {$[114]$} \\
\hline $\begin{array}{l}\text { Onchocerca } \\
\text { flexuosa }\end{array}$ & & NC_016172 & {$[112]$} \\
\hline Setaria digitata & Setariidae & NC_014282 & {$[63]$} \\
\hline $\begin{array}{l}\text { Gnathostoma } \\
\text { spinigerum }\end{array}$ & Gnathostomatidae & NC_027726 & {$[37]$} \\
\hline $\begin{array}{l}\text { Gnathostoma } \\
\text { doloresi }\end{array}$ & & NC_032073 & {$[38]$} \\
\hline $\begin{array}{l}\text { Gnathostoma } \\
\text { nipponicum }\end{array}$ & & NC_034239 & [38] \\
\hline $\begin{array}{l}\text { Heliconema } \\
\text { longissimum }\end{array}$ & Physalopteridae & NC_016127 & {$[2]$} \\
\hline $\begin{array}{l}\text { Gongylonema } \\
\text { pulchrum }\end{array}$ & Gongylonematidae & NC_026687 & [103] \\
\hline Spirocerca lupi & Thelazioidea & NC_021135 & {$[35]$} \\
\hline Thelazia callipaeda & & NC_018363 & {$[32]$} \\
\hline Trichinella spiralis $^{a}$ & Trichinellidae & NC_002681 & {$[50]$} \\
\hline Limulus polyphemus $^{\mathrm{a}}$ & Limulidae & NC_003057 & [104] \\
\hline
\end{tabular}

andicates outgroup

${ }^{b}$ The reference for this sequence is about Philometra carassii (Nematoda:

Philometridae). We presume that the name has been changed subsequently
Spirocerca and Gongylonema genera, was produced before using a smaller number of mitogenomes [32, 36]. Therefore it can be concluded with confidence that Spirocerca lupi is closely related to Gongylonema pulchrum and that it belongs to Gongylonematidae family.

Taxonomic position of the Gnathostoma genus, officially classified in the infraorder Gnathostomatomorpha of the suborder Spirurina [37], again proved to be contentious: in several studies using $18 \mathrm{~S}$ rRNA, Gnathostomatomorpha formed a separate clade basal to all Spirurida [1, 4, 34], whereas in both studies reporting Gnathostoma mitogenomes [37, 38], phylogenomic analyses placed it within the Ascaridomorpha order. In our study, however, it formed a sister clade with (Camallanidae + (Dracunculidae + Philometridae) $)$ within the Camallanina suborder. It is difficult to conclude which of these topologies are artefactual, as Ascaridomorpha were not included in our study. However, as Camallanina mitogenomes were not available for those two reports [37, 38], and as only amino acid sequences of protein-coding genes (PCGs) were used for their phylogenomic analyses, we suspect that the close relationship with Ascaridomorpha is an artefact likely to have been caused by long-branch attraction (LBA) [39, 40]. Amino acid sequences often exhibit too low variability in closely related species to allow a satisfactory level of phylogenetic resolution [11]. Furthermore, our results are much closer to the established phylogenetic position of Gnathostomatomorpha. Resolution of the intriguing taxonomic position of this infraorder thus warrants sequencing of further Camallanina mitogenomes, and inclusion of a much broader range of Nematoda families in future phylogenomic studies. An LBA artefact was also observed between the two outgroups used in this analysis: Limulus polyphemus (a basal arthropod) and Trichinella spiralis (a basal nematode), but it is not likely to have affected the topology of the Spirurida order.

\section{Genome size}

At 17901 bp in size, the mitogenome of $C$. cotti is by far the largest among the available Spirurida mitogenomes (Additional file 2). It is also exceptionally large among the chromadorean nematodes, whose mitogenomes are usually in the range between 13 and $15 \mathrm{~Kb}[9,10]$. Enoplean nematodes exhibit a much stronger heterogeneity in mitogenome size, and sizes around and over 20 $\mathrm{Kb}$ are not uncommon [9, 10, 13]. Although duplicated protein-coding genes are common in mitogenomes of some metazoan groups [41, 42], major variations in mitogenome sizes can usually be attributed to differences in the length of noncoding regions, rather than variations in gene content $[10,16]$. In agreement with this, the few known chromadorean mitogenomes larger 
than C. cotti, mostly found in plant-parasitic nematodes, possess abnormally lengthy non-coding regions that harbor tandemly repeated sequences [13, 43-45]. Large duplicated coding regions haven't been observed in chromadorean mitogenomes so far. The only comparable duplications, containing PCGs, have been observed in the enoplean family Mermithidae $[45,46]$. Thus, as a vertebrate-parasitic nematode exhibiting a large number of duplicated PCGs and tRNAs (Fig. 1), C. cotti is an exception not only among chromadoreans, but also among almost all nematodes.

\section{Genomic architecture}

Most Nematoda mitogenomes contain 12 PCGs, as atp 8 gene is missing in all Spirurida and other more derived nematode groups [32], 22 tRNAs and two rRNAs, but the total number of genes is relatively variable $(37.6 \pm 2.9)$ [10]. Nevertheless, with 46 (complete or pseudo) genes (Table 2), C. cotti mitogenome is (again) exceptional. All of these genes are encoded on the same strand, which is typical for Chromadorean mitogenomes $[9,10,13]$. Disregarding the duplicated genes, the mitogenome possesses all of the standard 12 PCGs and both rRNAs. However, despite our best efforts, which included careful manual searches within all intergenic regions (IGR) in the mitogenome, we could only identify 19 of the standard 22 tRNAs, with Leu1 ${ }^{(\mathrm{CUN})}$, Ser $2^{\text {(AGN) }}$ and Phe missing.

In order to attempt to understand the evolutionary history of the unique mitogenomic architecture of $C$. cotti, we have compared it to other available Spirurida mitogenomes (Fig. 1). Gene order is almost perfectly conserved within the Spirurina suborder; minor exceptions are Heliconema longissimum, where tRNA-V and tRNA-M have switched places, and two species where minor rearrangements within the standard A-L2-N-M-K tRNA box can be observed: Chandlerella quiscali (ML2-K-A-N) and Onchocerca volvulus (K-A-L2-N-M). As we suspected a possibility of an annotation artefact in $H$. longissimum ( $\mathrm{V}$ and $\mathrm{M}$ ), we have checked the two tRNAs: our results indicate that the genomic segment annotated as tRNA-Val [2] is not a functional tRNA. A deeper analysis of the entire mitogenome would be needed to determine the exact extent of its genomic architecture rearrangements.

In comparison to the Spirurina clade, genome architecture in the Camallanina clade is very different and very variable. A large number of gene rearrangements, including PCGs as well, can be observed, both between the two suborders and within the Camallanina clade. In terms of the order of PCGs (disregarding the tRNAs), three main patterns can be observed within the Camallanina clade: Gnathostomatidae pattern (perfectly conserved architecture among the three included species);
Dracunculidae + Philometridae pattern exhibiting a transposition of the fragment containing nad6, nad4l and $12 \mathrm{~S}$ rRNA genes and a large number of tRNA rearrangements (a large number of tRNA rearrangements can be also observed between the two included species, Philometroides sanguineus and Dracunculus medinensis); and finally the unique Camallanidae pattern exhibiting a large number of rearrangements in the order of PCGs, as well as PCG duplications. Whereas the mitogenomic gene order in vertebrates is almost invariant, in invertebrates it is relatively variable; however, this is mostly due to variation in the number and location of tRNAs, whereas the order of PCGs and rRNAs is considered to be stable [41]. Therefore, Camallanina clade is an intriguing outlier.

\section{Characteristics of duplicated fragments of the mitogenome}

To get a general idea about the origin and evolution of these duplicated fragments, we have compared the two nad5-A-Q-I-cytb-cox3-T-nad4 fragments (nad5-nad4 fragment henceforth). Because the two flanking genes (nad5-nad4) are not complete in both fragments (pseudogenes), only partial sequences of these two genes were included in the analysis. The upstream (in circular genomes this term can be ambiguous, so we use it here to refer specifically to the gene order as presented in Table 2) fragment was $2806 \mathrm{bp}$-long, and the downstream was $2767 \mathrm{bp}$; aligned, they were 2811 bp-long. The two fragments are highly conserved, with only 0.04 base substitutions per site between the two sequences. The difference in length was caused by the loss of 39 bases in the second fragment between positions 432 and 470 in the alignment. In the upstream fragment, the 39 bases are found in the intergenic region (73 bp) between pseudo-nad5 and tRNA-Ala, adjacent to the latter. The high similarity between the two segments indicates that they most probably originate from rearrangement events relatively recent in evolutionary terms.

\section{Characteristics of duplicated genes}

In the process of genomic rearrangements and/or subsequent sequence evolution several genes have lost fragments of their sequences, which is highly likely to have rendered them non-functional. We added a prefix 'pseudo-'to the names of those genes and did not indicate start/stop codons for them (Table 2). Among the six duplicated genes: nad1, nad4, nad5, nad2, cytb and cox3, only the latter three appear to possess two (mostly) complete copies. Both copies of cox3 use identical codons (ATT and TAA), are of identical length (762 bp), and have almost identical sequences, apart from five SNPs at positions 13, 29 and 109, 349, 398. Intriguingly, those translate into the equal number of mutations in the amino acid sequence, which is likely to be a sign of a relaxed purifying selection conferred by the functional 
Table 2 Organisation of the mitochondrial genome of Camallanus cotti

\begin{tabular}{|c|c|c|c|c|c|c|c|c|}
\hline \multirow[b]{2}{*}{ Gene } & \multicolumn{2}{|l|}{ Position } & \multirow[b]{2}{*}{ Size } & \multicolumn{2}{|c|}{ Codon } & \multirow[b]{2}{*}{ Anticodon } & \multirow[b]{2}{*}{ Strand } & \multirow[b]{2}{*}{$\mathrm{IG} / \mathrm{O}$} \\
\hline & Start & End & & Start & Stop & & & \\
\hline 125 & 1 & 714 & 714 & & & & + & \\
\hline tRNA-Trp-2 & 715 & 767 & 53 & & & TCA & + & \\
\hline tRNA-Asp & 767 & 824 & 58 & & & GTC & + & -1 \\
\hline pseudo-nad2 & 830 & 1640 & 811 & ATT & $\mathrm{T}-$ & & + & \\
\hline tRNA-Tyr & 1645 & 1698 & 54 & & & GTA & + & 4 \\
\hline pseudo-nad1 & 1701 & 2110 & 410 & & & & + & 2 \\
\hline pseudo-nad5 & 2390 & 2760 & 371 & & & & + & 279 \\
\hline pseudo-tRNA-Ala & 2834 & 2893 & 60 & & & TGC & + & 73 \\
\hline tRNA-GIn & 2883 & 2935 & 53 & & & TTG & + & -11 \\
\hline tRNA-Ile & 2934 & 2986 & 53 & & & GAT & + & -2 \\
\hline cytb & 2998 & 4081 & 1084 & $\mathrm{GTT}$ & $\mathrm{T}-$ & & + & 11 \\
\hline $\operatorname{cox} 3$ & 4126 & 4887 & 762 & ATT & TAA & & + & 44 \\
\hline tRNA-Thr & 4883 & 4937 & 55 & & & TGT & + & -5 \\
\hline nad4 & 4938 & 6170 & 1233 & $\mathrm{GTT}$ & TAG & & + & \\
\hline tRNA-Trp & 6170 & 6225 & 56 & & & TCA & + & -1 \\
\hline tRNA-Asn & 6226 & 6280 & 55 & & & $\mathrm{GTT}$ & + & \\
\hline tRNA-Glu & 6282 & 6336 & 55 & & & $\pi \mathrm{TC}$ & + & 1 \\
\hline pseudo-tRNA-Asp & 6337 & 6369 & 33 & & & - & & \\
\hline nad2 & 6370 & 7180 & 811 & ATT & $\mathrm{T}-$ & & + & \\
\hline tRNA-Tyr -2 & 7189 & 7242 & 54 & & & GTA & + & 8 \\
\hline nad1 & 7243 & 8118 & 876 & TTG & TAA & & + & \\
\hline tRNA-Lys & 8117 & 8176 & 60 & & & $\pi T$ & + & -2 \\
\hline tRNA-Leu2 ${ }^{\text {(UUR) }}$ & 8177 & 8233 & 57 & & & TAA & + & \\
\hline tRNA-Ser1 $1^{(\mathrm{UCN})}$ & 8234 & 8287 & 54 & & & TCT & + & \\
\hline atp6 & 8336 & 9002 & 667 & TTG & $\mathrm{T}-$ & & + & 48 \\
\hline $\operatorname{cox} 1$ & 9091 & 10,644 & 1554 & $\Pi \mathrm{TG}$ & TAG & & + & 88 \\
\hline tRNA-Cys & 10,646 & 10,700 & 55 & & & GCA & + & 1 \\
\hline tRNA-Met & 10,704 & 10,757 & 54 & & & CAT & + & 3 \\
\hline tRNA-Gly & 10,758 & 10,813 & 56 & & & TCC & + & \\
\hline $\operatorname{cox} 2$ & 10,814 & 11,489 & 676 & TTG & $\mathrm{T}_{-}$ & & + & \\
\hline tRNA-His & 11,493 & 11,543 & 51 & & & GTG & + & 3 \\
\hline AT-rich & 11,544 & 11,780 & 237 & & & & + & \\
\hline tRNA-Arg & 11,781 & 11,833 & 53 & & & ACG & + & \\
\hline $16 S$ & 11,881 & 12,818 & 938 & & & & + & 47 \\
\hline nad3 & 12,808 & 13,141 & 334 & $\Pi \mathrm{TG}$ & $T_{-}$ & & + & -11 \\
\hline nad5 & 13,142 & 14,725 & 1584 & $\pi \mathrm{G}$ & TAG & & + & \\
\hline tRNA-Ala & 14,750 & 14,803 & 54 & & & TGC & + & 24 \\
\hline tRNA-GIn - 2 & 14,808 & 14,860 & 53 & & & TTG & + & 4 \\
\hline tRNA-Ile-2 & 14,859 & 14,911 & 53 & & & GAT & + & -2 \\
\hline cytb-2 & 14,909 & 16,007 & 1099 & $\mathrm{GTT}$ & $\mathrm{T}-$ & & + & -3 \\
\hline $\cos 3-2$ & 16,052 & 16,813 & 762 & ATT & TAA & & + & 44 \\
\hline tRNA-Thr-2 & 16,809 & 16,863 & 55 & & & TGT & + & -5 \\
\hline pseudo-nad4 & 16,879 & 17,121 & 243 & & & & + & 15 \\
\hline
\end{tabular}


Table 2 Organisation of the mitochondrial genome of Camallanus cotti (Continued)

\begin{tabular}{|c|c|c|c|c|c|c|c|c|}
\hline \multirow[b]{2}{*}{ Gene } & \multicolumn{2}{|l|}{ Position } & \multirow[b]{2}{*}{ Size } & \multicolumn{2}{|c|}{ Codon } & \multirow[b]{2}{*}{ Anticodon } & \multirow[b]{2}{*}{ Strand } & \multirow[b]{2}{*}{$\mathrm{IG} / \mathrm{O}$} \\
\hline & Start & End & & Start & Stop & & & \\
\hline tRNA-Pro & 17,129 & 17,181 & 53 & & & TGG & + & 7 \\
\hline tRNA-Val & 17,181 & 17,239 & 59 & & & TAC & + & -1 \\
\hline nad6 & 17,238 & 17,673 & 436 & $\pi G$ & T- & & + & -2 \\
\hline nad4L & 17,668 & 17,901 & 234 & TTG & TAG & & + & -6 \\
\hline
\end{tabular}

IG/O: positive values indicate a non-coding intergenic segment, negative values indicate an overlap. Based on the hypothetical evolutionary history of genomic rearrangements (see Additional file 3 for details), we added a suffix ' -2 ' to the names of genes presumed to be copies of the original genes. Where sequence analysis indicated a loss of functionality, we added a prefix 'pseudo-'

redundancy. Pairwise comparison with related homologs showed that the cox3 copy (which we presume to be the original gene, Additional file 3A) appears to be the faster-evolving one. Regardless, both copies might still be functional.

Between the two $c y t b$ copies, the original one (cytb) lacks $15 \mathrm{bp}$ at the $5^{\prime}$ end (1099 vs $\left.1084 \mathrm{bp}\right)$. This is an annotation artefact caused by a different start codon choice: as opposed to the 3 bp overlap between $c y t b-2$ and the upstream flanking tRNA-Ile-2, there is an $11 \mathrm{bp}$ IGR between the $c y t b$ and tRNA-Ile (Table 2). Although the GTT start codon is conserved at the 3' end of tRNA-Ile, a deletion mutation in what is now the IGR between the two genes has caused a frameshift in the ORF of cytb. If this is not merely a sequencing artefact, the mutation may have rendered this (original) copy non-functional, or it simply uses the next GTT triplet as the start codon (as proposed in our annotation). Comparison with homologs indicates that several of them are merely one ATT triplet longer (the preceding triplet in C. cotti cytb is AAT), whereas Gnathostoma species even lack additional six amino acids at their 5' end. Otherwise, both sequences are relatively similar, with merely 0.04 base substitutions per site, but these translate to nine polymorphic sites between the two amino acid sequences.

Our analyses suggest that nad2 gene is likely to be a part of a relatively large tRNA-Asp + nad $1+$ tRNA-Tyr + nad2 duplicated fragment (Additional file 3). With 0.09 base substitutions per site, the two fragments appear to be relatively highly conserved, but translated protein sequences exhibit 0.15 amino acid substitutions per site, suggesting unusually high number of nonsynonymous mutations. Translated sequences revealed that the nad 2 copy, hypothesised by us to be the original gene (Additional file 3), possesses internal stop codons and thus is most probably non-functional. To reflect this, although the sequence is complete, we have added the prefix 'pseudo-'to its name (Table 2). At the end of the same fragment, we found a highly conserved 410 bases-long fragment of the $5^{\prime}$ end of nad1 gene, including a conserved TTG start codon. The rest of the gene is highly degraded or missing. Pseudo-nad4 is a relatively well-conserved 243 bp 5' fragment of the gene, whereas pseudo-nad5 is a highly degraded 3' fragment of the gene. The pattern of conserved/deleted fragments for all three genes is in agreement with the hypothetical evolutionary history of genomic rearrangements, with deletions at the edges of the copied fragments (Additional file 3). Therefore, we hypothesise that the losses of the ends of these genes may have occurred during the rearrangement events.

Sequence duplications are non-adaptive events [47] likely to reduce the evolutionary fitness of the organism, and thus should be under an evolutionary pressure directed towards the loss of the superfluous duplicated genes and genomic regions [48], although experimental evidence indicates that this process is not always very efficient [49]. Different levels of conservation between these six duplicated genes indicate either that the speed of this process of removal of superfluous genomic regions varies between different parts, or that rearrangement events occurred over a relatively long time-period.

\section{Transfer RNAs}

Non-standard secondary structures of tRNAs, usually lacking either a TYC or a DHU arm, are common in nematodes $[9,13,50,51]$. Duplicated tRNAs are also not particularly rare [10], but usually it is merely one tRNA that is duplicated, e.g. [27], whereas C. cotti mitogenome possesses seven pairs of duplicated tRNAs: Trp, Tyr, Ala, Asp, Gln, Ile, and Thr. On the other hand, nematodes usually possess all 22 standard tRNAs $[9,10]$, whereas we could identify only 19 , with tRNA-Leu $1^{(\mathrm{CUN})}, \operatorname{Ser} 2^{(\mathrm{AGN})}$ and tRNA-Phe missing. Generally, tRNAs are the gene category with the highest 'dispensability' in the mitochondrial genome, so mitogenomes of some animals don't possess the full set of tRNAs [10,52], which is believed to be compensated by the import of tRNAs from the cytoplasm [53]. We can only speculate that this may be the mechanism through which $C$. cotti compensates for the absence of these three tRNAs as well.

We have used the inferred hypothetical evolutionary history of genomic architecture rearrangements (Additional file 3) to attempt to search for traces of these missing tRNAs. Based on the gene orders in 
related species (Fig. 1), we expected tRNA-Leu1 ${ }^{(\mathrm{CUN})}$ to be found in the tRNA-Gln to nad4 duplicated fragment, between $c y t b$ and cox3 (Additional file 3). Indeed, a 44 bp-long IGR exists in both fragment copies in C. cotti (Table 2). Although the two IGRs are almost identical, with only $\mathrm{G}$ replaced with $\mathrm{T}$ at position 21 in the duplicated fragment, the alignment with tRNALeu1 ${ }^{(\mathrm{CUN})}$ homologs indicates low similarity, the absence of a conserved anticodon, and a large deletion at the $5^{\prime}$ end. The most probable position of tRNA-Phe would be downstream from tRNA-Arg, which is where we found a 47 bp IGR in $C$. cotti. The non-coding sequence did indeed exhibit similarity to related homologs, but a 6-bp deletion where the anticodon should be indicates that it has also lost its functionality. We presumed that tRNASer2 should be in the place where tRNA-Trp-2 is found in the mitogenome (Additional file 3). Sequence comparison has indeed confirmed that the two tRNA-Trp genes don't share evolutionary ancestry (Fig. 2), and that tRNA-Trp-2 is much more similar to tRNA-Ser2 homologs than to tRNA-Trp homologs. However, its anticodon base triplet has undergone a mutation from TGA to TCA. As a result, the mitogenome now possesses what appear to be two functional tRNA-Trp genes. Remolding of tRNAs is a well-documented process that involves a duplication of a tRNA gene, a mutation that changes the anticodon and the loss of the ancestral tRNA gene [54], but in this case there is no indication that tRNA-Ser2 was duplicated in the first place. Suspecting a sequencing artefact, we have checked the electropherogram, but there was no indication of poor sequencing quality either. This phenomenon should be further confirmed using a C. cotti specimen belonging to a different lineage.

Another indication of remolding was observed in tRNA-Val, as its sequence was very divergent from all remaining homologs, but the anticodon defined it as Valine. Comparison with other species indicates that its position is rather instable (Fig. 1). In the closely related D. medinensis, tRNA-Cys can be found in that position, but the sequence isn't similar to tRNA-Cys homologs either. BLASTing of the sequence did not return any significant hits either. We can only speculate that this phenomenon might be a reflection of another tRNA remolding in the evolutionary history of this species, where a mutation in the anticodon has led to subsequent rapid evolution of the entire DNA sequence.

The status of the tRNA-Ala-2, presumed to be a part of the duplicated fragment with pseudo-nad5 gene, is also ambiguous: its anticodon and central part of the

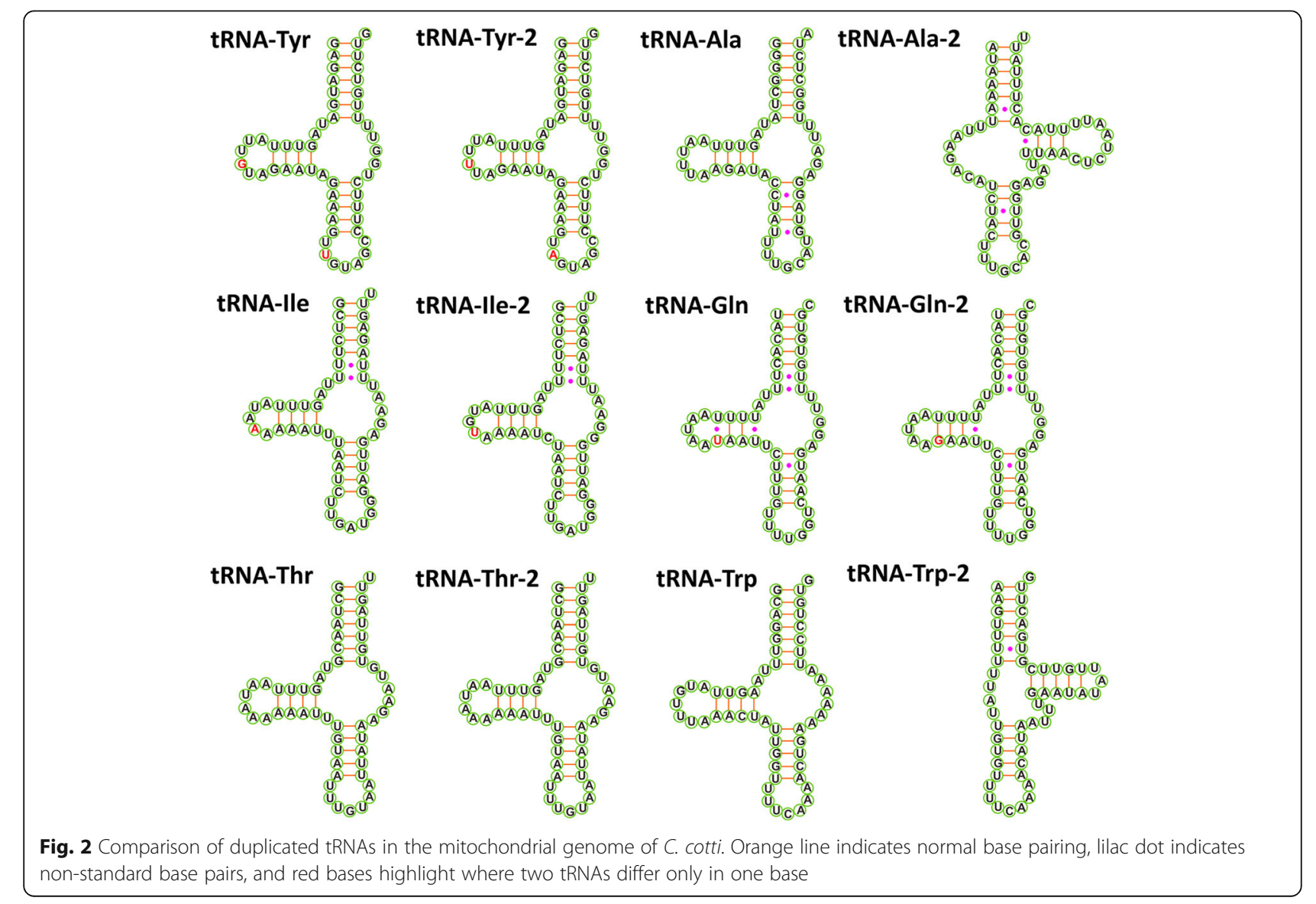


sequence are conserved among the related homologs, but its $5^{\prime}$ end exhibits a 6-bp deletion. It can still be folded into a tRNA-like structure, but a very nonstandard one, and very different from the tRNA-Ala copy (Fig. 2). Thus we remain doubtful regarding its functionality.

The annotation of tRNA-Lys was also ambiguous: depending on the algorithm employed, it was predicted as both Asn and Lys (ARWEN indicated it could be Lys or Asn, and MITOs that it is Asn). Comparison with homologs was also ambiguous, as the two most similar sequences appeared to be $D$. medinensis tRNA-Lys and $P$. sanguineus tRNA-Asn. As the anticodon was UUU, the tRNA was identified as Lys. Alignment with related homologs indicated that these two tRNAs (Lys and Asn) in Camallanoidea and Dracunculoidea families are generally very different from other related homologs, which suggests a possibility of yet another remolding event(s) in the common ancestor of these two families.

Although the annotation of tRNA-Pro was not ambiguous, it was the only homolog that exhibited GGA anticodon, whereas the remaining spirurids all use GGG. tRNA-Thr, tRNA-Gln and tRNA-Ile were duplicated as a part of the large tRNA-Gln - nad4 fragment (Additional file 3). The two tRNA-Thr sequences were identical, whereas the latter two pairs of sequences both exhibited only one SNP (Fig. 2). tRNA-Tyr and tRNAAsp were probably duplicated in a fragment also containing nad1 and nad2 (Additional file 3). However, possibly because tRNA-Tyr is located between the two PCGs, both copies are highly conserved, exhibiting only two SNPs and identical structure (Fig. 2). In contrast to this, one of the two tRNA-Asp copies has completely lost the $5^{\prime}$ part of its sequence (pseudo-tRNA-Asp in Table 2). The loss is likely to have occurred in the rearrangement process, as it is located on the edge of the copied fragment (Additional file 3). The rearrangement event is also likely to have been relatively recent in evolutionary terms, as the 33 bps adjacent to nad2 ( $3^{\prime}$ end) still exhibit a highly conserved sequence with only two polymorphic nucleotides in comparison to the corresponding fragment of the other tRNA-Asp copy.

\section{Base composition and skewness}

Mitogenomes of nematodes usually exhibit an $\mathrm{A}+\mathrm{T}$ bias, often higher than 70\% [35], and sometimes even higher than $80 \%$ [13]. Thus, despite its apparently high $\mathrm{A}+\mathrm{T}$ bias of $70.8 \%$, C. cotti actually exhibits the lowest bias among the available Spirurida mitogenomes, with the exception of Gnathostoma doloresi at 70.5\% [38]. This might be a reflection of lower mutational constraints on the non-functional duplicated parts of the mitogenome. Intriguingly, in Spirurida and Tylenchida orders, this bias mostly appears to be driven by the extremely high $\mathrm{T}$-content, with $\mathrm{T}$ base often representing more than $50 \%$ of the nucleotides [22]. Apart from the three Gnathostoma species and P. sanguineus, all available Spirurida mitogenomes exhibit T-bias values over $50 \%$ : from C. cotti at $51 \%$ to $56.9 \%$ in Dirofilaria repens (Additional file 2).

The studied mitogenome exhibits a negative AT-skew $(-0.441)$ and a positive GC-skew (0.463). In this aspect it is also not an outlier among the available spirurid mitogenomes, which exhibit AT-skews between -0.4 and -0.5 , and GC-skews between 0.35 and 0.52 (Additional file 2). In agreement with the T-base abundance findings, the three Gnathostoma species $(-0.28,-0.29$ and -0.33$)$ and $P$. sanguineus $(-0.21)$ exhibited lower AT-skew values. Surprisingly though, $H$. longissimum, which was not an outlier in terms of Tbase abundance (52.9\%), was an outlier in terms of ATskew (-0.34). Additional intriguing observation is that the two mitogenomes belonging to the outgroup species, L. polyphemus and T. spiralis, exhibited an opposite skew trend, with positive AT-skew and negative GCskew values.

\section{Overlaps between genes}

The thirteeen observed overlaps ranged from 1 to $11 \mathrm{bp}$ (Table 2), but we consider only five of them relatively large ( $\geq 5 \mathrm{bp}$ ): pseudo-tRNA-Ala/tRNA-Gln and 16S/

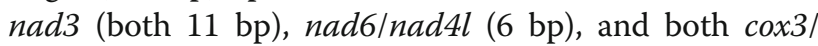
tRNA-Thr segments (5 bp). The first overlap is likely to be an annotation artefact caused by the previously discussed 6-bp deletion at the $5^{\prime}$ end of pseudo-tRNA-Ala. Although it may appear that the $16 S /$ nad3 overlap is also likely to be an annotation artefact, as the exact boundaries of rRNAs are difficult to predict and thus usually presumed to extend to adjacent genes [55], alignment of $16 \mathrm{~S}$ with other available spirurid homologs revealed that they are highly conserved, and that removing the overlap from the annotation would require truncating its relatively conserved $3^{\prime}$ end. As the C. cotti $16 \mathrm{~S}$ rRNA, at $938 \mathrm{bp}$, is already the smallest reported so far, with comparable sizes observed only in Gnathostomatidae (Additional file 2), we decided to keep the overlap. Furthermore, an 8 bp-long overlap with nad3 was also proposed in two other spirurids: S. lupi and Thelazia callipaeda [35]. The cox3/tRNA-Thr overlap, also perfectly preserved in the duplicated fragment, can be reduced down to only $3 \mathrm{bp}$ if cox3 uses the truncated $\mathrm{T}$ - stop codon, presumed to be completed (UAA) by the addition of 3' A residues to the mRNA [56-58]. Apart from nad6/nad4l, all of the overlaps in the studied mitogenome involve tRNA genes. This is common, and believed to be a consequence of lesser evolutionary constraints on tRNA sequences [59]. However, nad4l is a very small gene (234 bp in $C$. cotti), which also appears 
to be under lesser evolutionary constraints as mitogenomes of some groups of animals often exhibit overlaps involving this gene $[60,61]$. Thus we don't deem this overlap particularly suspicious. A large number of gene overlaps in mitochondrial genomes of metazoans are believed to be a reflection of strong selection for small size [19]. Although it may appear incongruous to observe as many as 13 gene overlaps in a mitogenome carrying such a large number of (presumably) unnecessary duplicated genes, they are most probably merely a remnant of a more stable (and compact) phase in the evolutionary history of this mitogenome.

\section{Protein-coding genes: Length and codons}

Gene lengths among the available Spirurida mitogenomes were not very conserved, with four genes exhibiting a difference of more than $50 \mathrm{bp}$ between the largest and the smallest compared gene: nad3 was the most conserved with only $16 \mathrm{bp}$ and nad2 the least with $228 \mathrm{bp}$ (Additional file 2). None of the C. cotti PCGs were major outliers regarding the size. Most of the genes used the two start (8 TTG + 3 ATT) and three stop (4 TAG, 3 TAA, $7 \mathrm{~T}-)$ codons common for nematodes [9, 13, 20, 27]. The third observed start codon, GTT for $c y t b$ (and $c y t b-2$ ) and nad4, was also observed in a number of closely related nematodes [38, 62, 63]. The length of nad4 remains ambiguous: we have selected the length of $1233 \mathrm{bp}$, identical to some Onchocerca species (Additional file 2), in which case there are no intergenic nucleotides between tRNAThr and nad4. On the other hand, as the $5^{\prime}$ end of the nad4 gene is GTTTTTTATGTTCTGTTT, the second GTT may also be used as the start codon, in which case there would be nine intergenic nucleotides and nad4 gene would be 1224 bp long, which is identical to G. doloresi.

\section{Non-coding regions}

The AT-rich region, believed to function as the control region containing the replication origin [64], is usually located between nad4 and tRNA-Met in mitogenomes of other nematodes [7]. In C. cotti these two genes are not adjacent and the AT-rich (237 bp) is located between tRNA-His and tRNA-Arg (Table 2). Notwithstanding the AT-rich, IGRs found in the mitogenome ranged from 1 to $279 \mathrm{bp}$ in length (21 in total). These numbers are larger (both the total number and maximum size) than reported in several closely related Spirurida mitogenomes: 14-16 IGRs, 1 to 62 bp [32, 35], which is not unexpected for a mitogenome exhibiting such a large number of rearrangements.

\section{Evolution of mitogenomic arschitecture}

Mitogenomic gene rearrangements should be strongly selected against, as they can affect the replication and transcription mechanisms, and produce disruptions in the gene expression co-regulation [65]. In agreement with this, although mitogenomes of nematodes exhibit exceptional variability in the mitogenomic architecture, most of it is found in several families within the class Enoplea, whereas Chromadorea (containing the majority of Nematoda species) exhibit very few gene rearrangements $[2,7,9,10,27,43,46,66]$. Frequent sequence rearrangements and long sequence duplications comparable to the ones found in the $C$. cotti mitogenome have been observed only in nematodes belonging to the Mermithidae family (Enoplea). Several species within this family exhibit hypervariability in conspecific size variation in mitogenome size $(19-34 \mathrm{~Kb})$, owing to long duplicated fragments of several $\mathrm{Kb}$, containing both PCGs and tRNAs, present in different copy numbers within individual mitochondrial genomes [45, 46, 67]. The proposed mechanism explaining this hypervariability is mitogenomic recombination $[68,69]$. Interspecific mitochondrial recombination has been observed in several animal lineages aside nematodes, including fishes [70] and birds [42], but it is believed to be accompanied by mitochondrial gene inversions (frequent exchange of the coding strand) [10]. Characteristics of the C. cotti mitogenome, especially the encoding of all genes on a single strand and almost identical sequences between some of the duplicated genes, suggest that interspecific recombination is an unlikely scenario. The most common mechanism of mitogenomic architecture rearrangements is believed to be slipped-strand mispairing mechanism leading to a tandem duplication and subsequent evolutionary loss of duplicated genes (TDRL) $[10,48,49,65,71,72]$. However, the observed architecture and the conducted CREX analysis imply that the mechanism would require several duplications of the entire genome followed by extensive losses (Supplementary file $3 \mathrm{C}$ ). As we did not observe traces of the latter process in the mitogenome (we would expect it to produce a much larger number of NCRs), we believe that transposals and duplications followed by transposals of duplicated fragments are a more parsimonious explanation for the observed mitogenomic architecture rearrangements (Additional file $3, \mathrm{~A}$ and $\mathrm{B}$ ). The evolution of gene orders mostly driven by transpositions has been proposed in ascomycetes as well [73]. A fourth hypothesis would be fragmentation of the "standard" singlecircle mitogenome into multipartite genomes, followed by subsequent re-organisation into a single circular molecule (we did not find any indications that the mitogenome is fragmented in C. cotti). Mitogenome fragmentation is common in higher plants $[74,75]$, and has occurred independently in some metazoans, including mesozoans [76], cnidaria [77], insects [78], rotifers $[79,80]$, as well as nematodes $[21,81]$. A very similar scenario, preceded by an ancestral mitogenome 
duplication, has been proposed before for another nematode, Globodera ellingtonae [81]. Although the relative abundance of pseudogenes is in agreement with this course of events $[81,82]$ and a single genome duplication is more parsimonious than a series of duplications required for the TDRL process, it remains hypothetical.

Regardless of the exact rearrangement mechanism, mitogenomic architecture of $C$. cotti is unique not only among Chromadorea, but also among almost all nematodes. Although it is believed that lengthy sequence duplications are absent from mitogenomes of chromadorean nematodes [46] and that only rearrangements in tRNA order are common [27], our analysis shows that neither of the propositions is true for the families clustering in the Camallanina clade (as defined in this study). As gene order instability has been observed in another chromadorean order, Oxyurida [66], this hints towards a possibility that some chromadorean orders (or more precisely specific groups of families within them) might be particularly prone to mitogenomic rearrangements.

Based on the observation of high variability in mitogenomic architecture in several phylogenetically distant groups, it has been proposed that the acceleration of the rate of genomic rearrangements in the evolutionary history of metazoans has occurred independently on several occasions [10]. It is known that mitochondrial genomes of some lineages of vertebrates are evolutionary dynamic even at short timescales [83, 84]. For example, different duplication mechanisms were observed in highly dynamic mitogenomes of some salamanders [84]. As genomic rearrangements are random evolutionary events [72], not driven by positive selection [47], and as there is evidence for positive correlation between compactness of genomes and structural stability [85], there is mounting evidence that the evolution of mitogenomic architecture is highly discontinuous. Therefore we hypothesise that, although mitogenomic rearrangements are generally strongly selected against, which results in long evolutionary periods of stasis in gene content and arrangement in most metazoan lineages, once a rearrangement event has destabilized the genomic architecture, this is likely to be followed by an exponentially accelerated rate of mitogenomic rearrangements.

\section{Conclusions}

We have sequenced and characterised the complete mitochondrial genome of the fish-parasitising chromadorean nematode Camallanus cotti. It is exceptionally large among chromadoreans and exhibits a unique architecture, with a large number of duplicated genes (46 genes and pseudogenes were identified in the mitogenome). Among the six duplicated PCGs, three were incomplete, and one contained stop codons in its sequence; thus only two pairs are likely to be functional.
Among the six duplicated tRNAs, five still appear to possess two functional copies. Regardless of the unusually large number of tRNAs found, only 19 unique ones were found in the mitogenome. A remolding event explains one missing tRNA, whereas intergenic regions provide clues for the other two tRNAs missing from the mitogenome. Although TDRL is considered to be the most common mechanism for gene order rearrangements, the most parsimonious explanation for the architecture observed would be either TDRL accompanied by transposals, or an even more unorthodox duplicationfragmentation-reassembly scenario.

Based on the observed rate of mitogenomic architecture rearrangements in Spirurida, as well as the evidence from other metazoans, we put forward a hypothesis that the evolution of mitogenomic architecture is highly discontinuous: once a long period of stasis in gene order and content has been punctuated by a rearrangement event, such a destabilised mitogenome is much more likely to undergo subsequent rearrangement events, resulting in an exponentially accelerated evolutionary rate of mitogenomic rearrangements. This hypothesis still needs to be tested using a large number of mitogenomes and appropriate statistical methods, but the implications of this model are particularly important for the gene order similarity analyses, which have often been used as an additive source of phylogenetic information for Chromadorea class $[2,10,27,43,46,66]$. The mounting evidence for discontinuous evolution of mitogenomic order in this class indicates that the aforementioned approach is too prone to overinflated estimates of evolutionary distance to have practical usability.

Chromadorean nematodes might be a good model to further study this discontinuity in the dynamics of mitogenomic evolution, as the class is comprised mostly of orders exhibiting extreme (for nematodes) stability of mitogenomic architecture, interrupted by clades of taxa that exhibit varying degrees of accelerated mitogenomic architecture evolution. As C. cotti possesses a mitogenome exhibiting an extremely accelerated rate of rearrangements, future studies should aim to sequence more mitogenomes belonging to families comprising the Camallanina clade, with particular focus on the Camallanidae family.

\section{Methods \\ Sampling}

It is known from a previous study that Chinese hooksnout carp (Opsariichthys bidens Günther, 1873) is particularly susceptible to $C$. cotti infections, with mean prevalence of almost $50 \%$ in wild populations from a large reservoir in central China, Danjiangkou (32 $82^{\prime} 50^{\prime \prime}$

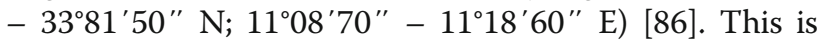
most probably in the native range of C. cotti [24]. 
Parasitic nematodes were obtained post mortem from hooksnout carp specimens caught by fishermen in the Danjiangkou and bought from the local market on 23/ Apr/2016. Live nematodes were removed from the fish intestines, and then taxonomically identified by their morphological characteristics via dissecting microscopy, using several sources as guidance [25, 87, 88]. All nematodes were washed in $0.6 \%$ saline before being stored in absolute ethanol in the Museum of Aquatic Organisms, Institute of Hydrobiology, Chinese Academy of Sciences, Wuhan, China (Accession No. IHB20160324005).

\section{Genome sequencing and assembly}

Genome was sequenced broadly following the procedure described before [60, 80]. After soaking a single adult nematode $(\approx 3.5 \mathrm{~cm})$ in TE buffer $(\mathrm{pH} 8.0)$ overnight to remove the ethanol, total genomic DNA was extracted using the Aidlab DNA extraction kit (Aidlab Biotechnologies, Beijing). Eight degenerate primer pairs were designed (Table 3 ) to match the generally conserved regions of mtDNA genes and used to amplify and sequence short fragments of $12 \mathrm{~S}$, cytb, nad4, nad1, cox1, cox2, 16S and nad5 genes. These sequences were then used to design 12 specific primers for the amplification and sequencing of the remaining mitogenomic sequences in several PCR steps. Primers were designed to produce amplicons with overlaps of about $100 \mathrm{bp}$. Reaction volume of $50 \mu \mathrm{L}$ contained $5 \mathrm{U} / \mu \mathrm{L}$ of TaKaRa LA Taq polymerase (TaKaRa, Japan), $10 \times$ LATaq Buffer II, $2.5 \mu \mathrm{M}$ of dNTP mixture, $0.2-1.0 \mu \mathrm{M}$ of each primer, 60 ng of DNA template, and PCR-grade $\mathrm{H}_{2} \mathrm{O}$. PCR conditions were optimized for each reaction, with the annealing temperature adjusted to suit the specific primer pair, extension time set to $1 \mathrm{~min}$ per $\mathrm{Kb}$ of the expected product size, and cycles (35 on average) adjusted depending on the amplification efficiency of primers. PCR products were sequenced on an ABI 3730 automatic sequencer using Sanger method [89]. When problems in sequencing were encountered, products were cloned into a pMD18-T vector (TaKaRa, Japan) and then sequenced. All obtained fragments were quality-proofed (electropherogram) and BLASTed [90] to confirm that the amplicon is the actual target sequence. Whenever the quality was sub-optimal, sequencing was repeated. Mitogenome was assembled stepwise with the help of DNAstar v7.1 [91] program, making sure that the overlaps were identical, and that no numts [28] were incorporated into the sequence. After it became obvious that the architecture of this mitogenome is very unusual, to further confirm that this is not a consequence of PCR artefacts, DNA pollution, numts, or a feature unique to the specimen from which the DNA was extracted, we have extracted DNA from another specimen (sampled as described above from the same fish
Table 3 Primers used for amplification and sequencing of the mitochondrial genome of C. cotti

\begin{tabular}{|c|c|c|}
\hline Gene span & Primer name & Sequence $\left(5^{\prime}-3^{\prime}\right)$ \\
\hline \multirow[t]{2}{*}{ nad6-12S } & DYF1 & TTGCGGTGCTTTGCGTTCTG \\
\hline & DYR1 & CTCTCGTTTAACAGTCAACC \\
\hline \multirow[t]{2}{*}{$12 S$} & XW12SF & GTTCCAGATTAATCGGCTA \\
\hline & XW12SR & CAATTGATGGATGATTTGTACC \\
\hline \multirow[t]{2}{*}{$125-c y t b$} & DYF2 & CACGAGTTAAGGTTGAGCCAC \\
\hline & DYR2 & CAACGTAGAGCAAGAAACC \\
\hline \multirow[t]{2}{*}{ cytb } & XWCYTBF & GRGCTCARATGAADTATTGAGC \\
\hline & XWCYTBR & TATCACTCTGGCACHAYATG \\
\hline \multirow[t]{2}{*}{ cytb-nad4 } & DYF3 & CCTTITCTGATGGGGGATCC \\
\hline & DYR3 & GAACACAACACAGCACCCAA \\
\hline \multirow[t]{2}{*}{ nad4 } & XWND4F & GCYCATGTTGARGCACCTAC \\
\hline & XWND4R & GAAGAATARGCAGCTAAWG \\
\hline \multirow[t]{2}{*}{ nad4-nad1 } & DYF4 & GTTATTGTCGTTITTGGGTGC \\
\hline & DYR4 & GGGTAATACAAATCACAACATCAG \\
\hline \multirow[t]{2}{*}{ nad1 } & XWND1F & GCGTRTTGGTCCTAAYAAGG \\
\hline & XWND1R & TATCAWAACGGWAACGTGGG \\
\hline \multirow[t]{2}{*}{ nad1-cox1 } & DYF5 & GTGTTCCTTTGTTCTACTC \\
\hline & DYR5 & GAACTTCCTGACAAACAACTAC \\
\hline \multirow[t]{2}{*}{$\operatorname{cox} 1$} & XWCOX1F & ATYGGTGGATTYGGWAATTG \\
\hline & XWCOX1R & TAAACCTCNGGGTGHCCAA \\
\hline \multirow[t]{2}{*}{$\operatorname{cox} 1-\cos 2$} & DYF6 & ACTATGTTGTTACTAGATCG \\
\hline & DYR6 & CCACAAGGCACCACACAACG \\
\hline \multirow[t]{2}{*}{$\cos 2$} & XWCOX2F & CTGAACTTATGATTACAGA \\
\hline & XWCOX2R & CCACAAATCTCWGAACAYTGACC \\
\hline \multirow[t]{2}{*}{ cox2-16S } & DYF7 & GGCAGATGCTTTGTCTGGGG \\
\hline & DYR7 & TTCCGAAGACTTATCTTG \\
\hline \multirow[t]{2}{*}{$16 \mathrm{~S}$} & XW16SF & ATGGCTGYTWTAGCGTGAGG \\
\hline & XW16SR & TCTATCTCACGAYGAAYTAAAC \\
\hline \multirow[t]{2}{*}{ 16S-nad5 } & DYF8 & GTAGTTCTTATACATTITCAGG \\
\hline & DYR8 & ATGGTCAACAGACCAACA \\
\hline \multirow[t]{2}{*}{ nad5 } & XWND5F & TAGCYTTAGGYAATCACCTAC \\
\hline & XWND5R & GAGACAWGGTNCTCAAWGCCAC \\
\hline \multirow[t]{2}{*}{ nad5 } & DYF9 & GGCTITTGTITTCTGATG \\
\hline & DYR9 & CTACATGCAAAATAGGTATC \\
\hline \multirow[t]{2}{*}{ nad5-cytb-2 } & DYF10 & GGTGCTGGATAGTTATTCTGG \\
\hline & DYR10 & CAATCAACTCAAAACAACAG \\
\hline \multirow[t]{2}{*}{ cytb-2-nad6 } & DYF11 & GTTCTGTTCCTAGGAAGATC \\
\hline & DYR11 & CCACAAAAACTCAACAAAGGAC \\
\hline \multirow[t]{2}{*}{ nad6 } & DYF12 & CTATGTTTCGCTTACAACG \\
\hline & DYF13 & CTACTCATAACACAAAAAC \\
\hline
\end{tabular}

Primer names beginning with XW indicate a generic primer, whereas DY indicates a specific primer pair 
specimen), and repeated the procedure using long-range PCR with the specific primers (Table 3 ).

For reasons we only partially understand, amplification and sequencing of this mitogenome was a comparatively difficult and laborious process, wherein obtaining both good quality PCR and sequencing results was not a straightforward process, especially for the duplicated segments. This was observed before for helminths, and suspected to be a result of high $\mathrm{A}+\mathrm{T}$ content [92]. Further elements contributing to this may include the low amount of DNA that can be obtained from a single nematode, or even complex quaternary structure of the sequences.

\section{Annotation}

The mitogenome was annotated broadly following the procedure described before [61, 93]. Protein-coding genes were found by searching for ORFs (employing genetic code 5, invertebrate mitochondrial) and alignment against the adopted reference genome ( $D$. medinensis) in Geneious [94]. Each gene was then aligned with homologous sequences of closely related species (Table 1) and annotation fine-tuned manually. The two ribosomal RNAs were annotated in a similar way, via comparison with homologs. MITOS [95] and ARWEN [96] tools were used to detect and fold the tRNAs in the genome. Folded tRNAs were further prepared for publication using Coreldraw (Corel Corp.). Because tRNAs of nematodes often exhibit non-standard secondary structures [50], after the automatic annotation by these algorithms, all non-coding regions larger than $30 \mathrm{bp}$ were carefully examined for the existence of tRNA-like sequences. Ambiguous tRNAs were additionally visually aligned with homologs extracted from all analysed mitogenomes using a custom-made GUI-based program MitoTool [97]. Annotation of tRNAs proved to very ambiguous, mostly as a result of many tRNA genes with non-standard sequences and/or secondary structures. We have tweaked the annotation several times, but we remain non-confident that it is fully accurate. MitoTool was also used to extract the annotation manually recorded in a Word document and to generate tables with statistics for all analysed mitogenomes. Pairwise distances were computed using MEGA 7 [98]. NCBI's Organelle Genome Resources were used to compare it with other published mitogenomes.

\section{Phylogenetic and gene order analyses}

Following the proposed guidelines for new mitogenome validation [30], we have conducted a barcoding identification assessment and a mitochondrial phylogenomic analysis using almost the entire new mitogenome. For the former, all 99 Camallanidae sequences were retrieved from the BOLD database [31]. Sequences were aligned by MAFFT [99], and raxmlGUI [100, 101] used to conduct maximum-likelihood phylogenetic analysis with 1000 bootstrap replications. For the phylogenomic analyses, all 21 available (June 2017) unique Spirurida mitogenomes were retrieved from the GenBank. It should be noted that sometimes Camallanida are elevated to a status of order [102], but the GenBank classification system includes them in Spirurida. Preliminary comparisons of mitogenomes retrieved from GenBank have indicated that Gongylonema pulchrum (NC_026687) [103] nad4 gene was misannotated. Hence, in order to be able to conduct phylogenetic and gene comparison analyses, we have re-annotated the gene by moving the start codon 15 bases in $5^{\prime}$ direction, and changing it from ATT to GTT, thus creating an overlap with the adjacent tRNAThr. In all analyses, two sequences were used as outgroups: a basal nematode [27, 43] T. spiralis (NC_002681) [50] and a basal arthropod L. polyphemus (NC_003057) [43, 104]. Complete mitogenomic sequences selected for the analysis were retrieved from the GenBank, genes extracted using MitoTool, and further handled with another custom-made GUI-based program, BioSuite [105]. Phylogenetic analyses were performed using concatenated 33 genes: 12 PCGs (atp 8 missing), 2 rRNAs and 19 tRNAs (three missing from $C$. cotti). For duplicated genes, the one presumed to be functional, or original where both seemed to be functional (see Table 2 and discussion for details), were used. Each sequence was aligned separately (in batches) by MAFFT integrated into BioSuite: rRNAs and tRNAs were aligned using normal alignment mode, whereas codon-alignment mode was used for PCGs. Finally, BioSuite was used to concatenate the alignments and produce input files for the two programs used to conduct the phylogenetic analysis. Maximum-likelihood analysis (with 1000 bootstrap replications) was conducted using raxmlGUI, and Bayesian inference using MrBayes 3.2.6 [106] with default settings and $5 \times 10^{6}$ generations (average SD of split frequencies $=0.000054) . \mathrm{GTR}+\mathrm{G}+\mathrm{I}$ evolution model, selected using ModelGenerator [107], was used in both analyses. Saturation analysis results, conducted using DAMBE program [108], indicate low saturation for all three codon positions. Finally, phylograms and gene orders were visualised and annotated by iTOL [109] with the help of several dataset files generated by MitoTool.

To make it easier for the readers to visualise the extent of rearrangements in the Camallanina clade, we present some of the hypothetical rearrangement scenarios in the Additional file 3 . However, these results should be interpreted with caution. Using the obtained BI phylogram, we conducted an ancestral character gene order state reconstruction using MLGO web server [110]. None of the available tools to infer genome rearrangements (among those we could find) could handle mitogenomes 
with both gene deletions and duplications. Therefore, we have inferred the approximate number of gene order rearrangements needed between Camallanus cotti and ancestral node A1, and shown them in the Additional file 3A. As the putative gene orders of the ancestral nodes A2 and A1 (as defined in Fig. 1) inferred by MLGO did not contain duplicated genes, CREX algorithm [111] was used to infer the hypothetical evolutionary history of gene order rearrangements (Additional file 3C). However, the results suggest three duplications of the entire mitogenome followed by a random loss of genes. As we could not find evidences supporting this scenario, i.e. numerous NCRs, we are skeptical about this result. Therefore, we have also visualised (Additional file $3 \mathrm{~B}$ ) the extent of gene order rearrangements between the two nodes following the same logic as in the Additional file 3A.

\section{Additional files}

Additional file 1: Taxonomic identification of the studied C. cotti nematode using cox 1 barcoding. Maximum likelihood analysis was conducted on 100 Camallanidae cox 1 sequences, with GenBank accession numbers shown in the figure. The clade containing the queried sequence ('Camallanus cotti this study') is shaded purple. (PDF $40 \mathrm{~kb}$ )

Additional file 2: Statistics and gene features of Spirurida mitogenomes. Worksheet A lists all the species, GenBank accession numbers, genome sizes in bp, base composition and skew. Worksheet B lists gene sizes for all species, and their corresponding (putative) start and terminal codons. Species are represented by acronyms of their binomial scientific names. (XLSX $18 \mathrm{~kb}$ )

Additional file 3: Mitogenomic gene order rearrangements in the Camallanina suborder. (A). A hypothetical outline of gene order rearrangements between Camallanus cotti and ancestral node A1. Ancestral node $A 1$ is shown in Fig. 1. Genes and mitogenome fragments presumed to have undergone rearrangements are highlighted by different colours. Hypothetical rearrangement mechanism is indicated on the left, and arrows are used to indicate the putative translocation pathways. Where the presumed mechanism is duplication + transposal, arrows indicate the positions of both fragments in the downstream genome, where the fragment presumed to be translocated is labelled with the letter $C$. Where colouring is insufficiently unambiguous, fragments undergoing a rearrangement event are additionally underlined. The order in which the events are shown is (mostly) random. A star is used to indicate a deletion and a thick blue arrow to indicate a tRNA remolding event. (B) A hypothetical outline of gene order rearrangements between ancestral nodes A2 and A1. Ancestral nodes are shown in Fig. 1. Genes and mitogenome fragments presumed to have undergone rearrangements are highlighted by different colours. Hypothetical rearrangement mechanism is indicated on the left, and arrows are used to indicate the putative translocation pathways. Where colouring is insufficiently unambiguous, fragments undergoing a rearrangement event are additionally underlined. The order in which the events are shown is (mostly) random. MLGO was used to infer the putative ancestral gene orders. (C). Hypothetical evolutionary history of gene order rearrangements between ancestral nodes A2 and A1 inferred by CREX algorithm. Ancestral nodes are shown in Fig. 1. Tdrl indicates a tandem duplication and random loss event, where a duplication event (not shown) is followed by the loss of some elements (orange-shaded). This results in the remaining copies (blue-shaded) being moved to the front. (PDF $514 \mathrm{~kb}$ )

\section{Abbreviations}

Bp: Base pair; IGR: Intergenic region; Kb: Kilobase; LBA: Long branch attraction; ORF: Open reading frame; PCGs: Protein-coding genes; SD: Standard deviation; SNP: Single-nucleotide polymorphism; TDRL: Tandem duplication and random loss

\section{Acknowledgments}

Lab technicians Tuo Du and Wei-Mu Xuan helped conduct a part of the molecular lab work. We'd like to thank Adam Casciaro for insightful discussions regarding the English language-related issues, and two anonymous reviewers for the time and expertise they have invested into reviewing our manuscript.

\section{Funding}

This study was funded by the Earmarked Fund for China Agriculture Research System (CARS-45-15), the National Natural Science Foundation of China (31572658), and the Major Scientific and Technological Innovation Project of Hubei Province (2015ABA045). The funders had no role in the design of the study, collection, analysis and interpretation of data, and in writing the manuscript.

\section{Availability of data and materials}

The datasets supporting the conclusions of this article are included within the article and its additional file, as well as in the GenBank repository under the accession number MF580344 [28].

\section{Authors' contributions}

$\mathrm{ZH}$ collected the samples, participated in the design of the study, lab work, data analysis, and helped write the manuscript; IJ participated in the design of the study, data analysis, data visualisation, and co-wrote the manuscript; $\mathrm{RC}$ participated in the molecular lab work, data analysis, and carried out sequence alignments; DZ contributed custom-made software and codes, and participated in sample collection, data analysis and data visualisation; $J Z$ participated in the design of the study and in the molecular lab work; GTW and WXL conceived and coordinated the study, and reviewed the manuscript. All authors contributed comments to the final version of the manuscript. All Authors read and approved the final manuscript.

\section{Ethics approval}

All experimental procedures involving animals were reviewed, approved and supervised by the Animal Care Committee of the Institute of Hydrobiology, Chinese Academy of Sciences. As the study did not involve any (live) vertebrates, nor regulated invertebrates, no special permits were required to retrieve and process the samples.

\section{Consent for publication}

Not applicable

\section{Competing interests}

The authors declare that they have no competing interests.

\section{Publisher's Note}

Springer Nature remains neutral with regard to jurisdictional claims in published maps and institutional affiliations.

\section{Author details}

${ }^{1}$ Key Laboratory of Aquaculture Disease Control, Ministry of Agriculture, and State Key Laboratory of Freshwater Ecology and Biotechnology, Institute of Hydrobiology, Chinese Academy of Sciences, Wuhan 430072, People's Republic of China. ${ }^{2}$ Bio-Transduction Lab, Wuhan Institute of Biotechnology, Wuhan 430075, People's Republic of China. ${ }^{3}$ University of Chinese Academy of Sciences, Beijing 100049, People's Republic of China.

Received: 4 August 2017 Accepted: 24 October 2017 Published online: 02 November 2017

\section{References}

1. Černotíková E, Horák A, Moravec F. Phylogenetic relationships of some spirurine nematodes (Nematoda: Chromadorea: Rhabditida: Spirurina) parasitic in fishes inferred from SSU rRNA gene sequences. Folia Parasitol (Praha). 2011;58:135-48.

2. Park J-K, Sultana T, Lee S-H, Kang S, Kim HK, Min G-S, et al. Monophyly of clade III nematodes is not supported by phylogenetic analysis of complete mitochondrial genome sequences. BMC Genomics. 2011;12:1-16.

3. Koutsovoulos GD. Reconstructing the phylogenetic relationships of nematodes using draft genomes and transcriptomes: University of Edinburgh, PhD Thesis. Edinburgh: University of Edinburgh, PhD Thesis; 2015. 
4. Meldal BHM, Debenham NJ, De Ley P, De Ley IT, Vanfleteren JR, Vierstraete $A R$, et al. An improved molecular phylogeny of the Nematoda with special emphasis on marine taxa. Mol Phylogenet Evol. 2007;42:622-36.

5. van Megen $\mathrm{H}$, van den Elsen $\mathrm{S}$, Holterman M, Karssen $\mathrm{G}$, Mooyman $\mathrm{P}$, Bongers T, et al. A phylogenetic tree of nematodes based on about 1200 full-length small subunit ribosomal DNA sequences. Nematology. 2009;11:927-50.

6. Poinar $\mathrm{G}$. Trends in the evolution of insect parasitism by nematodes as inferred from fossil evidence. J Nematol. 2003;35:129-32.

7. Liu G-H, Shao R, Li J-Y, Zhou D-H, Li H, Zhu X-Q. The complete mitochondrial genomes of three parasitic nematodes of birds: a unique gene order and insights into nematode phylogeny. BMC Genomics. 2013;14:414.

8. Duchêne S, Archer FI, Vilstrup J, Caballero S, Morin PA. Mitogenome phylogenetics: the impact of using single regions and partitioning schemes on topology, substitution rate and divergence time estimation. PLoS One. 2011;6:e27138

9. Hu M, Gasser RB. Mitochondrial genomes of parasitic nematodes-progress and perspectives. Trends Parasitol. 2006:22:78-84

10. Gissi C, lannelli F, Pesole G. Evolution of the mitochondrial genome of Metazoa as exemplified by comparison of congeneric species. Heredity (Edinb). 2008;101:301-32062.

11. Cameron SL. Insect mitochondrial genomics: implications for evolution and phylogeny. Annu Rev Entomol. 2014;59:95-117.

12. Nelson LA, Lambkin CL, Batterham P, Wallman JF, Dowton M, Whiting MF et al. Beyond barcoding: a mitochondrial genomics approach to molecular phylogenetics and diagnostics of blowflies (Diptera: Calliphoridae). Gene. 2012:511:131-42.

13. Sun L, Zhuo K, Lin B, Wang H, Liao J. The complete mitochondrial genome of Meloidogyne Graminicola (Tylenchina): a unique gene arrangement and its phylogenetic implications. PLoS One. 2014;9:e98558.

14. Andersson SGE, Karlberg O, Canbäck B, Kurland CG. On the origin of mitochondria: a genomics perspective. Philos. Trans. R. Soc. B biol. Sci. 2003; 358:165-79.

15. Sagan L. On the origin of mitosing cells. J Theor Biol. 1967;14:225-IN6.

16. Boore JL. Animal mitochondrial genomes. Nucleic Acids Res. 1999;27:1767-80.

17. Rand DM. "Why genomes in pieces?" revisited: sucking lice do their own thing in mtDNA circle game. Genome Res Cold Spring Harbor Lab Press. 2009;19:700-2.

18. Taanman J-W. The mitochondrial genome: structure, transcription, translation and replication. Biochim. Biophys. Acta - Bioenerg. 1999;1410:103-23.

19. Wolstenholme DR. Animal mitochondrial DNA: structure and evolution. Int Rev Cytol. 1992;141:173-216.

20. Okimoto R, Macfarlane JL, Wolstenholme DR. Evidence for the frequent use of TTG as the translation initiation codon of mitochondrial protein genes in the nematodes, Ascaris suum and Caenorhabditis Elegans. Nucleic Acids Res. 1990;18:6113-8.

21. Armstrong MR, Blok VC, Phillips MS. A multipartite mitochondrial genome in the potato cyst nematode Globodera pallida. Genetics. 2000;154:181-92.

22. Gibson T, Farrugia D, Barrett J, Chitwood DJ, Rowe J, Subbotin S, et al. The mitochondrial genome of the soybean cyst nematode, Heterodera glycines. Genome. 2011;54:565-74

23. Wu S-G, Wang G-T, Xi B-W, Gao D, Nie P, Wu SG, et al. Molecular characteristics of Camallanus Spp. (Spirurida: Camallanidae) in fishes from China based on its rDNA sequences. J Parasitol. 2008;94:731-6.

24. Wu S, Wang G, Gao D, Xi B, Yao W, Liu M. Occurrence of Camallanus Cotti in greatly diverse fish species from Danjiangkou reservoir in central China. Parasitol Res. 2007;101:467-71.

25. Levsen A, Berland B. The development and morphogenesis of Camallanus Cotti Fujita, 1927 (Nematoda: Camallanidae), with notes on its phylogeny and definitive host range. Syst Parasitol. 2002;53:29-37.

26. Su Y-B, Kong S-C, Wang L-X, Chen L, Fang R. Complete mitochondrial genome of Philometra carassii (Nematoda: Philometridae). Mitochondrial DNA Taylor \& Francis. 2016;27:1397-8

27. Kim J, Lee SH, Gazi M, Kim T, Jung D, Chun JY, et al. Mitochondrial genomes advance phylogenetic hypotheses for Tylenchina (Nematoda: Chromadorea). Zool Scr BioMed Central. 2015;44:446-62.

28. Hazkani-Covo E, Zeller RM, Martin W. Molecular poltergeists: mitochondrial DNA copies (numts) in sequenced nuclear genomes. PLoS Genet. 2010;6(2): e1000834. https://doi.org/10.1371/journal.pgen.1000834.

29. Kikuchi T, Afrin T, Yoshida M. Complete mitochondrial genomes of four entomopathogenic nematode species of the genus Steinernema. Parasit Vectors. 2016;9:430.
30. Botero-Castro F, Delsuc F, Douzery EJP. Thrice better than once: quality control guidelines to validate new mitogenomes. Mitochondrial DNA. 2014; 1736:1-6.

31. Ratnasingham S, Hebert PDN. A DNA-based registry for all animal species: the barcode index number (BIN) system. PLoS One. 2013;8:e66213.

32. Liu GH, Gasser RB, Otranto D, Xu MJ, Shen JL, Mohandas N, et al. Mitochondrial genome of the Eyeworm, Thelazia callipaeda (Nematoda: Spirurida), as the first representative from the family Thelaziidae. PLoS Negl Trop Dis. 2013;7:1-9.

33. Smythe AB, Sanderson MJ, Nadler SA. Nematode small subunit phylogeny correlates with alignment parameters. Syst Biol. 2006;55:972-92.

34. Nadler SA, Carreño RA, Mejía-Madrid H, Ullberg J, Pagan C, Houston R, et al. Molecular phylogeny of clade III nematodes reveals multiple origins of tissue parasitism. Parasitology. 2000;134:1421-42.

35. Liu G-H, Wang Y, Song H-Q, Li M-W, Ai L, Yu X-L, et al. Characterization of the complete mitochondrial genome of Spirocerca lupi: sequence, gene organization and phylogenetic implications. Parasit Vectors. 2013;6:45.

36. Yilmaz E, Fritzenwanker M, Pantchev N, Lendner M, Wongkamchai S, Otranto D, et al. The mitochondrial genomes of the Zoonotic canine filarial parasites Dirofilaria (Nochtiella) repens and Candidatus Dirofilaria (Nochtiella) Honkongensis provide evidence for presence of cryptic species. Makepeace BL, editor. PLoS Negl Trop Dis.; 2016;10:e0005028.DE GRUYTER.

37. Liu G-H, Shao R, Cai X-Q, Li W-W, Zhu X-Q. Gnathostoma Spinigerum mitochondrial genome sequence: a novel gene arrangement and its Phylogenetic position within the class Chromadorea. Sci Rep Nature Publishing Group. 2015;5:12691.

38. Sun M-M, Ma J, Sugiyama H, Ando K, Li W-W, Xu Q-M, et al. The complete mitochondrial genomes of Gnathostoma doloresi from China and Japan. Parasitol. Res. Springer. Berlin Heidelberg. 2016;115:4013-20.

39. Bergsten J. A review of long-branch attraction. Cladistics. 2005;21:163-93.

40. Graybeal A. Is it better to add taxa or characters to a difficult phylogenetic problem? Syst Biol. 1998;47:9-17.

41. Breton S, Milani L, Ghiselli F, Guerra D, Stewart DT, Passamonti M. A resourceful genome: updating the functional repertoire and evolutionary role of animal mitochondrial DNAs. Trends Genet. 2014;30:555-64.

42. Sammler S, Bleidorn C, Tiedemann R. Full mitochondrial genome sequences of two endemic Philippine hornbill species (Aves: Bucerotidae) provide evidence for pervasive mitochondrial DNA recombination. BMC Genomics. 2011;12:35.

43. Sultana T, Kim J, Lee S-H, Han H, Kim S, Min G-S, et al. Comparative analysis of complete mitochondrial genome sequences confirms independent origins of plant-parasitic nematodes. BMC Evol Biol. 2013;13:1-17.

44. Humphreys-Pereira DA, Elling AA. Mitochondrial genomes of Meloidogyne Chitwoodi and M. Incognita (Nematoda: Tylenchina): comparative analysis, gene order and phylogenetic relationships with other nematodes. Mol. Biochem. Parasitology. 2014;194:20-32.

45. Azevedo JLB, Hyman BC. Molecular characterization of lengthy mitochondrial DNA duplications from the parasitic nematode Romanomermis culicivorax. Genetics. 1993;133:933-42.

46. Hyman BC, Lewis SC, Tang S, Wu Z. Rampant gene rearrangement and haplotype hypervariation among nematode mitochondrial genomes. Genetica. 2011;139:611-5.

47. Marlétaz F, Le Parco Y, Liu S, Peijnenburg KT. Extreme Mitogenomic variation in natural populations of Chaetognaths. Genome Biol Evol. 2017;9: 1374-84. Oxford University Press, Oxford

48. San Mauro D, Gower DJ, Zardoya R, Wilkinson M. A hotspot of gene order rearrangement by tandem duplication and random loss in the vertebrate mitochondrial genome. Mol Biol Evol. 2006;23:227-34.

49. Shi W, Gong L, Wang S-Y, Miao X-G, Kong X-Y. Tandem duplication and random loss for mitogenome rearrangement in Symphurus (Teleost: Pleuronectiformes). BMC Genomics. 2015;16:355.

50. Lavrov DV, Brown WM. Trichinella spiralis mtDNA: a nematode mitochondrial genome that encodes a putative ATP8 and normally structured tRNAs and has a gene arrangement relatable to those of Coelomate metazoans. Genetics. 2001;157(2):621-37.

51. Jacob JEM, Vanholme B, Van Leeuwen T, Gheysen G. A unique genetic code change in the mitochondrial genome of the parasitic nematode Radopholus Similis. BMC Res Notes. 2009;2:192.

52. Lavrov DV, Pett W. Animal mitochondrial DNA as we do not know it: Mtgenome organization and evolution in Nonbilaterian lineages. Genome biol. Evolution. 2016;8:2896-913. 
53. Duchêne AM, Pujol C, Maréchal-Drouard L. Import of tRNAs and aminoacyltRNA synthetases into mitochondria. Curr Genet. 2009:55(1):1-18.

54. Sahyoun AH, Hölzer M, Jühling F, Höner Zu Siederdissen C, Al-Arab M, Tout $\mathrm{K}$, et al. Towards a comprehensive picture of alloacceptor tRNA remolding in metazoan mitochondrial genomes. Nucleic Acids Res. 2015;43:8044-56.

55. Kilpert F, Podsiadlowski L. The complete mitochondrial genome of the common sea slater, Ligia Oceanica (Crustacea, isopoda) bears a novel gene order and unusual control region features. BMC Genomics. 2006;7:241.

56. Temperley RJ, Wydro M, Lightowlers RN, Chrzanowska-Lightowlers ZM Human mitochondrial mRNAs-like members of all families, similar but different. Biochim Biophys Acta - Bioenerg Elsevier. 2010;1797:1081-5.

57. Ojala D, Montoya J, Attardi G. tRNA punctuation model of RNA processing in human mitochondria. Nature. 1981;290:470-4.

58. Schuster G, Stern D. RNA Polyadenylation and decay in mitochondria and chloroplasts. Prog Mol Biol Transl Sci. 2009:85:393-422.

59. Doublet V, Ubrig E, Alioua A, Bouchon D, Marcade I, Marechal-Drouard L. Large gene overlaps and tRNA processing in the compact mitochondrial genome of the crustacean Armadillidium vulgare. RNA Biol. 2015;12:1159-68.

60. Wen HB, Cao ZM, Hua D, Xu P, Ma XY, Jin W, et al. The complete maternally and paternally inherited mitochondrial genomes of a freshwater mussel Potamilus Alatus (Bivalvia: Unionidae). Waller RF, editor. PLoS One Public Lib Sci. 2017;12:e0169749.

61. Wang J-G, Zhang D, Jakovlić I, Wang W-M. Sequencing of the complete mitochondrial genomes of eight freshwater snail species exposes pervasive paraphyly within the Viviparidae Family (Caenogastropoda). PLoS One Public Lib Sci (PLoS). 2017;12:e0181699.

62. Hu M, Gasser RB, Abs El-Osta YG, Chilton NB. Structure and organization of the mitochondrial genome of the canine heartworm, Dirofilaria immitis. Parasitology. 2003;127:37-51.

63. Yatawara L, Wickramasinghe S, Rajapakse RPVJ, Agatsuma T. The complete mitochondrial genome of Setaria Digitata (Nematoda: Filarioidea): mitochondrial gene content, arrangement and composition compared with other nematodes. Mol Biochem Parasitol. 2010;173:32-8.

64. Bernt M, Braband A, Schierwater B, Stadler PF. Genetic aspects of mitochondrial genome evolution. Mol Phylogenet Evol. 2013;69:328-38.

65. Boore JL. The duplication/random loss model for gene rearrangement exemplified by mitochondrial genomes of Deuterostome animals. Netherlands: Springer; 2000. p. 133-47.

66. Kang S, Sultana T, Eom KS, Park YC, Soonthornpong N, Nadler SA, et al. The mitochondrial genome sequence of Enterobius Vermicularis (Nematoda: Oxyurida) - an idiosyncratic gene order and phylogenetic information for chromadorean nematodes. Gene. 2009;429:87-97.

67. Tang S, Hyman BC. Mitochondrial genome haplotype hypervariation within the isopod parasitic nematode Thaumamermis cosgrovei. Genetics. 2007; 176:1139-50.

68. Lunt DH, Hyman BC. Animal mitochondrial DNA recombination. Nature. 1997;387:247.

69. Lunt DH, Kumar S, Koutsovoulos G, Blaxter ML. The complex hybrid origins of the root knot nematodes revealed through comparative genomics. PeerJ. 2014;2:e356

70. Ciborowski KL, Consuegra S, García de Leániz C, Beaumont MA, Wang J, Jordan WC. Rare and fleeting: an example of interspecific recombination in animal mitochondrial DNA. Biol Lett. 2007;3:554-7.

71. Dowton M, Austin AD. Evolutionary dynamics of a mitochondrial rearrangement hot spot in the hymenoptera. Mol Biol Evol. 1999;16:298-309.

72. Wei S, Shi M, He J, Sharkey M, Chen X. The complete mitochondrial genome of Diadegma semiclausum (hymenoptera: ichneumonidae) indicates extensive independent evolutionary events. Genome. 2009:52:308-19.

73. Duò A, Bruggmann R, Zoller S, Bernt M, Grünig CR. Mitochondrial genome evolution in species belonging to the Phialocephala fortinii s.L. - Acephala applanata species complex. BMC Genomics. 2012;13:166.

74. Wu Z, Cuthbert JM, Taylor DR, Sloan DB. The massive mitochondrial genome of the angiosperm Silene Noctiflora is evolving by gain or loss of entire chromosomes. Proc Natl Acad Sci U S A. 2015;112:10185-91.

75. Kitazaki K, Kubo T, Kitazaki K, Kubo T. Cost of having the largest mitochondrial genome: evolutionary mechanism of plant mitochondrial genome. J Bot. 2010;2010:1-12.

76. Watanabe Kl, Bessho Y, Kawasaki M, Hori H. Mitochondrial genes are found on minicircle DNA molecules in the mesozoan animal Dicyema. J Mol Biol. 1999;286:645-50.
77. Pont-Kingdon G, Vassort CG, Warrior R, Okimoto R, Beagley CT, Wolstenholme DR. Mitochondrial DNA of Hydra Attenuata (Cnidaria): a sequence that includes an end of one linear molecule and the genes for I-rRNA, tRNA (f-met), tRNA (Trp), COll, and ATPase8. J Mol Evol. 2000;51:404-15.

78. Shao R, Kirkness EF, Barker SC. The single mitochondrial chromosome typical of animals has evolved into 18 minichromosomes in the human body louse, Pediculus humanus. Genome Res. 2009;19:904-12.

79. Suga K, Mark Welch DB, Tanaka Y, Sakakura Y, Hagiwara A. Two circular chromosomes of unequal copy number make up the mitochondrial genome of the rotifer Brachionus Plicatilis. Mol Biol Evol. 2008;25:1129-37.

80. Nie ZJ, Gu RB, Du FK, Shao NL, Xu P, Xu GC. Monogonont rotifer, Brachionus Calyciflorus, possesses exceptionally large, fragmented mitogenome. Yue B-S, editor. PLoS One Pub Lib Sci. 2016;11:e168263.

81. Phillips WS, Brown AMV, Howe DK, Peetz AB, Blok VC, Denver DR, et al. The mitochondrial genome of Globodera ellingtonae is composed of two circles with segregated gene content and differential copy numbers. BMC Genomics. 2016;17:706.

82. Wei DD, Shao R, Yuan ML, Dou W, Barker SC, Wang JJ. The multipartite mitochondrial genome of liposcelis bostrychophila: insights into the evolution of mitochondrial genomes in bilateral animals. PLoS One. 2012;7

83. Fujita MK, Boore JL, Moritz C. Multiple origins and rapid evolution of duplicated mitochondrial genes in Parthenogenetic geckos (Heteronotia Binoei; Squamata, Gekkonidae). Mol Biol Evol. 2007;24:2775-86. Sinauer Associates, Sunderland (MA)

84. Mueller RL, Boore JL. Molecular mechanisms of extensive mitochondrial gene rearrangement in Plethodontid salamanders. Mol Biol Evol. 2005;22: 2104-12. Oxford University Press

85. Lagisz M, Poulin R, Nakagawa S. You are where you live: parasitic nematode mitochondrial genome size is associated with the thermal environment generated by hosts. J Evol Biol. 2013;26:683-90.

86. Wu SG, Wang GT, Xi BW, Gao D, Nie P. Population dynamics and maturation cycle of Camallanus Cotti (Nematoda: Camallanidae) in the Chinese hooksnout carp Opsariichthys Bidens (Osteichthyes: Cyprinidae) from a reservoir in China. Vet Parasitol. 2007;147:125-31.

87. Menezes RC, Tortelly R, Tortelly-Neto R, Noronha D, Pinto RM. Camallanus Cotti Fujita, 1927 (Nematoda, Camallanoidea) in ornamental aquarium fishes: pathology and morphology. Mem Inst Oswaldo Cruz. 2006;101:683-7.

88. Wu S-G. Population biology and molecular ecology of the nematode Camallanus Cotti. Doctoral dissertation (in Chinese): Chinese Academy of Sciences. Chinese Academy of Sciences; 2007.

89. Sanger F, Nicklen S, Coulson AR. DNA sequencing with chain-terminating inhibitors. Proc Natl Acad Sci. 1977;74:5463-7.

90. Altschul SF, Madden TL, Schäffer AA, Zhang J, Zhang Z, Miller W, et al. Gapped BLAST and PSI-BLAST: a new generation of protein database search programs. Nucleic Acids Res. 1997;25:3389-402.

91. Burland TG. DNASTAR's Lasergene Sequence Analysis Software. In: Misener S., Krawetz S.A. (eds) Bioinformatics Methods and Protocols. Methods in Molecular Biology ${ }^{\mathrm{m}}$. Totowa: Humana Press. 2000;132:71-91. https://doi.org/ 10.1385/1-59259-192-2:71. ISBN 978-1-59259-192-3.

92. Hu M, Jex AR, Campbell BE, Gasser RB. Long PCR amplification of the entire mitochondrial genome from individual helminths for direct sequencing. Nat Protoc. 2007;2:2339-44.

93. Zhang D, Zou H, Wu SG, Li M, Jakovlić I, Zhang J, et al. Sequencing, characterization and phylogenomics of the complete mitochondrial genome of Dactylogyrus Lamellatus (Monogenea: Dactylogyridae). J Helminthol. 2017:1-12.

94. Kearse M, Moir R, Wilson A, Stones-Havas S, Cheung M, Sturrock S, et al. Geneious basic: an integrated and extendable desktop software platform for the organization and analysis of sequence data. Bioinformatics. 2012;28:1647-9.

95. Bernt $M$, Donath $A$, Jühling F, Externbrink F, Florentz C, Fritzsch G, et al. MITOS: Improved de novo metazoan mitochondrial genome annotation. Mol Phylogenet Evol. 2013;69:313-9.

96. Laslett D, Canbäck B. Arwen: a program to detect tRNA genes in metazoan mitochondrial nucleotide sequences. Bioinformatics. 2008;24:172-5.

97. Zhang D. MitoTool [Internet]. [cited 2017 Jun 26]. Available from: https://github.com/dongzhang0725/MitoTool.

98. Kumar S, Stecher G, Tamura K. MEGA7: molecular evolutionary genetics analysis version 7.0 for bigger datasets. Mol Biol Evol. 2016;33:1870-4.

99. Katoh K, Standley DM. MAFFT multiple sequence alignment software version 7: improvements in performance and usability. Mol Biol Evol. 2013; 30:772-80. 
100. Silvestro D, Michalak I. RaxmIGUI: a graphical front-end for RAxML. Org Divers Evol. 2012;12:335-7.

101. Stamatakis A. RAxML version 8: a tool for phylogenetic analysis and post-analysis of large phylogenies. Bioinformatics. 2014;30:1312-3.

102. ITIS. Integrated Taxonomic Information System (ITIS) [Internet]. [cited 2017 May 8]. Available from: http://www.tis.gov.

103. Liu G-H, Jia Y-Q, Wang Y-N, Zhao G-H, Zhu X-Q. The complete mitochondrial genome of the gullet worm Gongylonema pulchrum: gene content, arrangement, composition and phylogenetic implications. Parasit Vectors. 2015;8:100.

104. Lavrov DV, Boore JL, Brown WM. The complete mitochondrial DNA sequence of the horseshoe crab Limulus Polyphemus. Mol. Biol. Evolution. 2000;17:813-24.

105. Zhang D. Biosuite [Internet]. [cited 2017 Jun 26]. Available from: https://github.com/dongzhang0725/BioSuite.

106. Ronquist F, Huelsenbeck JP. MrBayes 3: Bayesian phylogenetic inference under mixed models. Bioinformatics. 2003;19:1572-4.

107. Keane TM, Creevey CJ, Pentony MM, Naughton TJM, Mclnerney JO. Assessment of methods for amino acid matrix selection and their use on empirical data shows that ad hoc assumptions for choice of matrix are not justified. BMC Evol Biol. 2006;6:29.

108. Xia X, Xie Z. DAMBE: software package for data analysis in molecular biology and evolution. J Hered. 2001;92:371-3.

109. Letunic I, Bork P. Interactive tree of life (iTOL): an online tool for phylogenetic tree display and annotation. Bioinformatics. 2007;23:127-8.

110. Hu F, Lin Y, Tang J. MLGO: phylogeny reconstruction and ancestral inference from gene-order data. BMC Bioinformatics. 2014;15:354.

111. Bernt M, Merkle D, Ramsch K, Fritzsch G, Perseke M, Bernhard D, et al. CREx: inferring genomic rearrangements based on common intervals. Bioinformatics. 2007;23:2957-8.

112. McNulty SN, Vaughan JA, Tkach W, Mullin AS, Weil GJ, Fischer PU, Comparing the mitochondrial genomes of Wolbachia-dependent and independent filarial nematode species. BMC Genomics. 2012;13:145.

113. Ghedin E, Wang S, Spiro D, Caler E, Zhao Q, Crabtree J, et al. Draft genome of the filarial nematode parasite Brugia malayi. Science. 2007;317:1756-60.

114. Keddie EM, Higazi T, Unnasch TR. The mitochondrial genome of Onchocerca volvulus: sequence, structure and phylogenetic analysis. Mol Biochem Parasitol. 1998;95:111-27.

\section{Submit your next manuscript to BioMed Central and we will help you at every step:}

- We accept pre-submission inquiries

- Our selector tool helps you to find the most relevant journal

- We provide round the clock customer support

- Convenient online submission

- Thorough peer review

- Inclusion in PubMed and all major indexing services

- Maximum visibility for your research

Submit your manuscript at www.biomedcentral.com/submit

) Biomed Central 